\title{
Editorial
}

\section{Aquichan: una década de rigor y dedicación}

\author{
María Elisa Moreno-Fergusson \\ Profesora Asociada de la Facultad de Enfermería y Rehabilitación de la Universidad de La Sabana, \\ Colombia. Editora de la revista Aquichan. mariae.moreno@unisabana.edu.co
}

A quichan, voz de la cultura chibcha que significa "cuidar", fue el nombre escogido por las directivas de la Facultad de Enfermería y Rehabilitación de la Universidad de La Sabana, en Colombia, para denominar la revista de Enfermería cuyo primer número se publicó en el mes de octubre de 2001, en conmemoración del décimo aniversario del programa.

El vocablo Aquichan, entonces, sintetiza el interés de esta disciplina y, por tanto, refleja la esencia del quehacer profesional de enfermería. La misión de la revista es la de "ser un medio de expresión de la Facultad, orientado hacia la promoción y el estímulo del desarrollo teórico de la disciplina, así como de los principios y valores que orientan la Facultad”. Esta misión es loable, no solo porque el propósito de una publicación científica es dar visibilidad y promover el desarrollo del conocimiento en un campo del saber, sino porque el Programa de Enfermería de la Universidad de La Sabana fue el primero en Colombia en adoptar un modelo conceptual como marco de referencia del currículo.

En este sentido, desde el principio la Facultad de Enfermería asumió el reto de comprender e innovar la aplicación de la teoría de la disciplina en los diferentes ámbitos de la práctica profesional que, también, se materializó en el proyecto editorial Aquichan. Durante los primeros cinco años la publicación fue anual y el equipo editorial estuvo conformado por directivos y profesores del Programa de Enfermería, un representante de los egresados y profesionales colombianas y extranjeras reconocidas por su trayectoria.

De esta manera, Aquichan dio sus primeros y exógenos pasos en la literatura científica; caminar en el que siempre ha contado con la asesoría de una persona vinculada a la Oficina de Publicaciones de La Sabana, experta en el proceso editorial y conocedora de las formalidades de clasificación en los diferentes servicios de indexación y resumen (Sires), lo cual ha sido fundamental para garantizar el rigor, la calidad y la visibilidad.

En los números uno y dos los autores fueron profesores de la Facultad que publicaron aspectos importantes de la vida de la misma, como los curriculares y pedagógicos, entre los que se destacan la educación como proceso holístico; la enseñanza de la ética, los valores y las virtudes, y el proceso de autoevaluación para la acreditación de los programas. Entre los artículos relacionados con la investigación y con el desarrollo epistemológico de enfermería se destacan aspectos culturales del cuidado, y la publicación de manuscritos relacionados con el Modelo de Adaptación de Callista Roy y la aplicación de este en la práctica, hecho que puso al alcance de los profesionales de enfermería hispanoamericanos esta teoría.

A partir del 2003, el prestigio de Aquichan 
trasciende las fronteras y la participación de autores extranjeros supera la local y nacional, evidenciando así el reconocimiento como publicación científica de la disciplina. Fue entonces cuando el equipo editorial, bajo la dirección de María Clara Quintero Laverde, decana de la Facultad y hoy vicerrectora académica de La Sabana, optó por publicar resultados de investigación en inglés, en portugués y, desde luego, en español.

Estos desarrollos, sumados al aprovechamiento de las TIC, hicieron que en el 2005 Aquichan tuviera su versión on line e iniciara en firme su ruta de indexación presentándose a los Sires reconocidos nacional e internacionalmente. Primero se sometió al examen del Servicio Nacional de Indexación y Homologación - Publindex. Después fue indexada en otras bases bibliográficas, como Lilacs, Dialnet, Scielo, Cuiden, Redalyc, Fuente Académica-Ebsco, Hinari, Hela, Periódica y EMCareElsevier.

Por la mismas época, la revista fue incluida en los directorios más conocidos por la comunidad académica mundial como Ulrich's, DOAJ y Latindex. Google Académico es otra de las grandes ventanas de Aquichan, desde donde se pueden consultar y descargar sus contenidos.

Gracias a la aceptación por parte de la comunidad científica internacional; al trabajo objetivo, estricto y preciso de los evaluadores; a la calidad editorial, a la visibilidad y a la certificación por parte de las diferentes bases bibliográficas, en el 2007 Aquichan cambió su periodicidad y se convirtió en una revista semestral.

Todos estos avances, sumados a la estabilidad y al carácter mundial de los artículos publicados, permitieron que en el 2010 Aquichan fuera indexada en el ISI Web of Knowledge, de
Thomson Reuters, y aceptada en Scopus, de la casa editora Elsevier. En este último índice los contenidos serán visibles en los próximos meses, como oficialmente se ha informado por parte del equipo editorial de Elsevier.

Esta cualificación de Aquichan ha traído consigo una mayor demanda no solo de sus lectores, sino de nuevos y habituales autores que ven en la revista de Enfermería de La Sabana un escenario real y efectivo para poner al alcance de la comunidad científica sus aportes al desarrollo disciplinar. Por esto, y también como respuesta a las exigencias de los Sires, hoy Aquichan circula tres veces al año: en abril, agosto y diciembre.

Estar indexado en algunos de los mejores índices del mundo implica estímulos y desafíos. De los primeros queda el registro con cada nuevo autor y en cada concepto que firman acreditados evaluadores, así como en las estadísticas de consultas reportadas por las diferentes bases bibliográficas, la cuales hablan de un promedio mensual de nueve mil quinientas.

De los segundos tenemos el reto de la citación: que los registros bibliográficos de artículos publicados en las revistas científicas de enfermería más destacadas den cuenta de lo publicado en Aquichan; que en cada nueva edición podamos comprobar los avances en productividad, sin bajar la calidad científica y editorial.

Estos retos nos motivan para continuar con nuestra misión de divulgar el conocimiento de enfermería y buscar los medios para que esté al alcance de los profesionales en todo el mundo y, de esta manera, incentivar su estudio y aplicación para promover el desarrollo de la disciplina y la calidad del cuidado que se brinda a los pacientes. 